\title{
Original
}

\section{Factores de riesgo cardiovascular en un grupo de mujeres con sobrepeso y obesidad}

\author{
(Cardiovascular risk factors among women with over \\ weight and obesity)
}

Viviana Esquivel-Solís, Marco Vinicio-Alvarado, Gina Solano-Quirós, Adriana Ramírez- Leandro

\section{Resumen}

Escuela de Nutrición. Facultad de Medicina. Universidad de Costa Rica.

Abreviaturas: AGB, Área grasa braquial; EBAIS, Equipos Básicos de Salud; ECV, enfermedad cardiovascular; HDL, high density lipoprotein (lipoproteínas de alta densidad); LDL, low density lipoprotein,(li poproteínas de baja densidad); NCEP, National Cholesterol Education Program (programa nacional de colesterol Estados Unidos); OMS, organización Mundial de la Salud; PAIS, programa de Atención Integral en Salud; VET, valor energético total.

Correspondencia:

Viviana Esquivel Solís.

Email: vesquive@nutricion.ucr. ac.cr
Justificación y objetivo: La enfermedad isquémica cardiovascular es definida por la Organización Mundial de la Salud como la incapacidad cardiaca aguda y crónica, resultante de la reducción o suspensión del flujo sanguíneo del miocardio, asociado con procesos patológicos en el sistema arterial coronario. Es una de las principales causas de muerte entre los individuos adultos de Costa Rica y el mundo. El presente trabajo describe los factores de riesgo de enfermedad cardiovascular hallados en un grupo de mujeres con sobrepeso y obesidad residentes del área de atracción de salud del programa de Atención Integral en Salud (PAIS), correspondientes a los distritos de La Unión, Curridabat y Montes de Oca.

Métodos: Se estudiaron un total de 90 mujeres diagnosticadas con sobrepeso u obesidad, con edades comprendidas entre los 18-49 años, 30 de cada uno de los distritos que conforman el área de cobertura PAIS (Curridabat, La Unión y Montes de Oca) durante 2007. Se evaluaron los factores de riesgo cardiovascular recomendados por el ATPIII establecidos por la Asociación Americana del Corazón.

Resultados: Un 75\% de las mujeres del estudio mostraron niveles de LDL colesterol fuera del rango aceptable, el $50 \%$ presentó niveles no deseables del colesterol total y el $51.3 \%$ presentó hipertensión. Los factores de riesgo predisponentes más prevalentes fueron la obesidad (64\%) y el $61 \%$ gastó menos de $2000 \mathrm{Kcal}$ por semana en actividad física que las clasifica como sedentarias. El único factor de riesgo condicionante que se evaluó fue la hipertrigliceridemia, presentada en el $25.3 \%$ de las mujeres.

Conclusión: Las mujeres en estudio presentaron al menos dos factores de riesgo cardiovascular; son un grupo de especial atención, de manera que sean sujeto de intervenciones de tipo integral que aborden la promoción de la salud, tanto en las mujeres como en sus familias y comunidades.

Descriptores: factores de riesgo, enfermedad cardiovascular, mujer.

\section{Abstract}

Background and aim: Ischemic heart disease is defined by the World Health Organization as an acute and chronic cardiac disability that results from the reduction of blood flow to the 
ISSN 0001-6002/2008/50/4/213-220 Acta Médica Costarricense, (C2008 Colegio de Médicos y Cirujanos myocardium. This is associated with pathological processes of the arterial coronary artery system and to the main cause of general mortality of adults in Costa Rica and the world. The present study describes the risk factors for cardiovascular disease found in a group of overweight and obese women, residents of the area under the Comprehensive Health Program (PAIS), a joint program of to the University of Costa Rica and the Social Security System.

Methods: We studied 90 overweight or obese women, aged 18-49 years, 30 women from each of the districts covered by PAIS (Curridabat, La Union and Montes de Oca), during 2007. The risk factors evaluated were all the ones recommended by ATPIII of the American Heart Association.

Results: Seventy four percent of women showed LDL cholesterol values off the acceptable range, $50 \%$ had non-desirable total cholesterol levels, $51.3 \%$ showed high blood pressure. More prevalent predisposing risk factors were obesity (64\%), and $61 \%$ expended less than $2000 \mathrm{Kcal}$ per week in physical activities, which classified them as sedentary. The only conditional risk factor that was evaluated was hypertrygliceridemia wich appeared in $25.3 \%$ of the women.

Conclusions: Women residents in the area covered by PAIS showed at least 2 cardiovascular risk factors. This group should be addressed with health promotion and preventive strategies in order to improve their own their family health and the community where they live.

Key words: risk factors, cardiovascular disease, women.

Recibido: 8 de noviembre de 2007

Aceptado: 29 de julio de 2008
Las enfermedades cardiovasculares son, desde la década del 70, la principal causa de muerte en Costa Rica. Dos cuartas partes se deben al infarto de miocardio y una cuarta parte a los accidentes cerebrovasculares. ${ }^{1,2}$ Para 1998, la principal causa de muerte para ambos sexos en el grupo de 15 a 34 años fueron las lesiones traumáticas y envenenamientos, seguida por los tumores en hombres y enfermedades cardiovasculares en las mujeres. En el grupo de 35 a 49 años la principal causa de muerte en las mujeres fueron los tumores $(42 \%)$, seguida por las enfermedades cardiovasculares y sus causas. ${ }^{3}$ En 2000 , la tasa de mortalidad por enfermedades del aparato circulatorio fue de 12.4 por 10 000 habitantes, de modo que las enfermedades cardiovasculares son un importante problema de salud en el país, especialmente en las mujeres. ${ }^{4}$

La causa principal de la enfermedad isquémica cardiovascular es la aterosclerosis, que se define como la acumulación subintimal focal de lípidos, glúcidos complejos, sangre y productos calcáreos acompañados de otros cambios en la capa media arterial, que obstruyen parcial o completamente el flujo de sangre en las arterias, provocando que el corazón no reciba oxígenoy sufra daño isquémico. ${ }^{5} \mathrm{El}$ desarrollo de aterosclerosis se asocia con diversos factores de riesgo como: antecedentes familiares de cardiopatía coronaria, sedentarismo, fumado, hipertensión arterial, diabetes, colesterol elevado y obesidad.

Desde 1991, las autoridades nacionales de salud han alertado sobre las características aterogénicas de la dieta del costarricense. ${ }^{6}$ El consumo de grasa en la población general se incrementó de un $20.0 \%$ en 1966 a un $27.4 \%$ en 1996 , y alcanza el $35 \%$ en los estratos socioeconómicos altos. Las altas concentraciones de ácidos grasos trans están asociadas con el aumento en el riesgo de sufrir un infarto agudo del miocardio, no fatal en adultos costarricenses. ${ }^{7,8}$ Otra característica de la dieta del costarricense que constituye un factor de riesgo aterógeno es la baja ingesta de fibra. ${ }^{9}$

En el 2001 se realizó en el área urbana del cantón central de Cartago un estudio denominado CARMEN (Conjunto de Acciones para la Reducción Multifactorial de las Enfermedades No Transmisibles), donde se encontró que la población practica estilos de vida poco saludables, caracterizados por una dieta inadecuada, sedentarismo y un alto consumo de alcohol y tabaco. Todo esto conduce a sobrepeso y obesidad, diabetes y padecimiento de enfermedades crónicas. ${ }^{10}$

La presente investigación tiene como objetivo describir los factores de riesgo cardiovasculares detectados en un grupo de mujeres con sobrepeso y obesidad residentes del área de atracción de salud del programa de Atención Integral en Salud (PAIS) de los distritos Curridabat, La Unión y Montes de Oca.

\section{Materiales y métodos}

Este estudio forma parte de la investigación "Característicasnutricionales, socioeconómicas,psicológicas y de estilo de vida en mujeres obesas en edad fértil". El 
estudio fue de tipo descriptivo transversal; en él se detalla el comportamiento de variables dietéticas, antropométricas, bioquímicas y clínicas en un tiempo determinado. Se trabajó con un total de 90 mujeres, 30 en cada uno de los distritos que conforman el área de cobertura PAIS (Curridabat, La Unión y Montes de Oca), con edades comprendidas entre los 18-49 años, diagnosticadas todas con sobrepeso y obesidad. Se excluyeron aquellas mujeres cuya obesidad estaba relacionada a trastornos fisiopatológicos como: hipertiroidismo, Síndrome Prader-Willi, enfermedad de Cushing, Diabetes Mellitus, entre otros.

Las variables estudiadas fueron los factores de riesgo cardiovascular definidos con los criterios establecidos por la Asociación Americana del Corazón, ${ }^{5}$ los cuales son: factores de riesgo mayores o causales de enfermedad cardiovascular, factores predisponentes de la enfermedad cardiovascular, factores condicionantes de la enfermedad cardiovascular y factores dietarios asociados a riesgo de enfermedad cardiovascular.

Todas las participantes residían en el área de atracción del Programa de Atención en Salud PAIS. Un 59\% reside en la provincia de San José y el 34\% en la provincia de Cartago. Un 34\% de las participantes reside en el cantón de Curridabat, un 34\% reside en el cantón de la Unión, 31\%, en Montes de Oca.

Las participantes recibieron una capacitación previa para el llenado de los formularios, específicamente el de actividad física y el registro de consumo de alimentos de tres días de Chinnock, A, ${ }^{11-13}$ el cual debía abarcar el listado total de alimentos consumidos durante dos días entre semana y un día de fin de semana.

La evaluación clínica fue realizada por un médico internista e incluyó la consulta de la presencia de antecedentes familiares y personales, antecedentes de sobrepeso, así como un examen físico completo.

En cuanto a la evaluación antropométrica, se midió la circunferencia braquial, el pliegue cutáneo tricipital, el peso, la talla y la circunferencia de cintura. La toma de medidas antropométricas se realizó en el laboratorio de Nutrición Humana de la Escuela de Nutrición de la Universidad de Costa Rica, con equipo previamente calibrado.

Como parte de las variables bioquímicas, se evaluó la glicemia en ayunas y el perfil lipídico a un total de 83 mujeres. Las muestras de sangre fueron tomadas en el EBAIS correspondiente a cada sujeto y analizadas en el laboratorio clínico de la Oficina de Salud de la Universidad de Costa Rica.

Todos los datos fueron analizados mediante el programa EP INFO 6 para la evaluación de la dieta y el programa SPSS 11.0. El análisis de las variables incluyó técnicas de estadística descriptiva.

\section{Resultados}

El promedio de edad del grupo en estudio fue de 37.99 \pm 8.63 años, con un rango de edad que va de los 21 hasta los 58 años. Respecto al estado civil de las participantes, la mayoría (71.1\%) está casada y el $70 \%$ son amas de casa. Al consultarles respecto al último grado de enseñanza aprobado, solo el $28.9 \%$ de las mujeres completó la primaria y el $22.4 \%$ logró terminar la educación secundaria.

\section{A. Factores mayores o causales de enfermedad cardiovascular}

Un total de 83 mujeres se realizaron las pruebas bioquímicas del perfil lipídico. De acuerdo con la clasificación del NCEP (Programa Nacional de Colesterol) (Estados Unidos), 2002, el $46.7 \%$ posee niveles de colesterol total deseables, solo el $23 \%$ pose niveles óptimos de LDL colesterol y el $20 \%$ tiene niveles bajos de HDL colesterol Cuadro 1.

Al evaluar los niveles de glicemia en ayunas, el 89.16\% presentó niveles normales; el $9.6 \%$ presentó intolerancia a los carbohidratos y el 1,2\% Diabetes Mellitus, según los valores de normalidad establecidos por American Diabetes Association, 2005.

\section{Fumado}

Dieciocho participantes indicaron ser fumadoras activas. De acuerdo con la clasificación de OMS, 1998, 10 son

\begin{tabular}{|c|c|c|}
\hline \multicolumn{3}{|c|}{$\begin{array}{c}\text { Cuadro 1. Distribución de la muestra según } \\
\text { niveles de colesterol total, colesterol LDL y } \\
\text { colesterol HDL * }\end{array}$} \\
\hline Categoría & Frecuencia & Porcentaje \\
\hline \multicolumn{3}{|l|}{ Colesterol total } \\
\hline Deseable $(<200 \mathrm{mg} / \mathrm{dl})$ & 42 & 50.6 \\
\hline Normal alto $(200-239 \mathrm{mg} / \mathrm{dl}$ & 29 & 34.9 \\
\hline Alto (> $240 \mathrm{mg} / \mathrm{dl})$ & 12 & 14.5 \\
\hline \multicolumn{3}{|l|}{ LDL-Colesterol } \\
\hline óptimo (<100 mg/dl) & 21 & 25.3 \\
\hline $\begin{array}{l}\text { Cercano/encima del óptimo }(100-129 \\
\mathrm{mg} / \mathrm{dl})\end{array}$ & 30 & 36 \\
\hline Normal alto (130-159 mg/dl) & 25 & 30 \\
\hline Alto (160-189 mg/dl) & 4 & 4.8 \\
\hline Muy alto (> 190 mg/dl) & 3 & 3.5 \\
\hline \multicolumn{3}{|l|}{ HDL Colesterol } \\
\hline Bajo (< 40 mg/dl) & 18 & 21.7 \\
\hline Normal $(40-60$ mg/dl) & 53 & 63.8 \\
\hline Ideal (> $60 \mathrm{mg} / \mathrm{dl})$ & 12 & 14.4 \\
\hline
\end{tabular}

*Valores de normalidad establecidos por NCEP 2002. 
fumadoras leves $(<$ de 5 cigarrillos por día), 6 moderadas (6-15 cigarrillos por día) y 2 severas (> 16 cigarrillos por día).

\section{Hipertensión arterial}

Según la valoración médica realizada a las mujeres del estudio, solo 39 presentaron niveles de presión arterial normal, conforme los criterios de NCEP, 2002 Cuadro 2.

\section{B. Factores predisponentes de enfermedad cardiovascular obesidad}

El 35\% de las mujeres presentaron sobrepeso y el $64 \%$, algún grado de obesidad, según el índice de masa corporal (IMC), siendo el sobrepeso y la obesidad grado I las predominantes Cuadro 4.

Al analizar el Área Grasa Braquial (AGB) según las tablas de Frisancho, se observa que de las mujeres con sobrepeso, el $56 \%$ tiene un exceso evidente de grasa y un $25 \%$ presenta tendencia al exceso. De igual forma, en las mujeres con obesidad, la mayoría presenta exceso evidente de grasa: un $80.6 \%$ de las participantes con obesidad grado I, un $89.5 \%$ de las que presentan obesidad grado II y $100 \%$ de aquellas que presentan obesidad grado III Cuadro 4 .

\section{Obesidad abdominal}

De las 90 encuestadas, $88(97.8 \%)$ presentan una circunferencia de cintura mayor a $88 \mathrm{~cm}$, lo que las clasifica según la OMS, 2000, con obesidad abdominal.

\section{Sedentarismo}

Según el índice de actividad física (11), el 61.1\% tiene un gasto menor a $2000 \mathrm{kcal}$ por semana, por lo que se clasifican como sedentarias.

\section{Historia de enfermedad cardiovascular prematura}

El $48.7 \%$ de las mujeres reportó tener antecedentes familiares de enfermedad cardiovascular, definidos como la
Cuadro 2. Distribución de la muestra según categoría de presión arterial

\begin{tabular}{|c|c|c|}
\hline Categoría (PAS/PAD)* & Frecuencia & Porcentaje \\
\hline No hipertensa & 39 & 48.7 \\
\hline Normal-alta $(130-139 / 85-89 \mathrm{~mm} \mathrm{Hg})$ & 11 & 13.8 \\
\hline $\begin{array}{l}\text { Hipertensión grado I (140-159/90-99 } \\
\mathrm{mmHg} \text { ) }\end{array}$ & 12 & 15 \\
\hline $\begin{array}{l}\text { Hipertensión grado II (160-179/100- } \\
\text { 109mmHg) }\end{array}$ & 12 & 15 \\
\hline $\begin{array}{l}\text { Hipertensión grado III (>180/>110 } \\
\mathrm{mmHg}\end{array}$ & 6 & 7.5 \\
\hline Total & 80 & 100 \\
\hline
\end{tabular}

*Según NCEP, 2002

\begin{tabular}{|lcc|}
\hline \multicolumn{3}{|c|}{$\begin{array}{c}\text { Cuadro 3. Distribución de la muestra según } \\
\text { Índice de Masa Corporal * }\end{array}$} \\
\hline \multicolumn{1}{|c|}{ Índice de Masa Corporal } & Frecuencia & Porcentaje \\
\hline Sobrepeso $25-29.9 \mathrm{~kg} / \mathrm{m}^{2}$ & 32 & 35.6 \\
Obesidad grado I $30-34.9 \mathrm{~kg} / \mathrm{m}^{2}$ & 31 & 34.4 \\
Obesidad grado II $35-39.9 \mathrm{~kg} / \mathrm{m}^{2}$ & 19 & 21.1 \\
Obesidad grado III $\geq 40 \mathrm{~kg} / \mathrm{m}^{2}$ & 8 & 8.9 \\
Total & 90 & 100 \\
\hline
\end{tabular}

* Clasificación según OMS, 2002

presencia de algún episodio de cardiopatía isquémica en un familiar de primer grado, ocurrido antes de los 65 años de edad en las mujeres y los 55 años en los hombres.

C. Factores condicionantes de enfermedad cardiovascular

\begin{tabular}{|c|c|c|c|c|c|c|c|c|c|}
\hline \multicolumn{10}{|c|}{ Cuadro 4. Distribución del área grasa braquial, según Índice de Masa Corporal } \\
\hline \multirow{4}{*}{$\begin{array}{l}\text { Categoría según Área } \\
\text { grasa braquial }\end{array}$} & \multicolumn{9}{|c|}{ Categoría según IMC } \\
\hline & \multirow{2}{*}{\multicolumn{2}{|c|}{$\begin{array}{c}\text { Sobrepeso } \\
\left(25-29.9 \mathrm{~kg} / \mathrm{m}^{2}\right)\end{array}$}} & \multicolumn{6}{|c|}{ Obesidad } & \multirow{3}{*}{ Total } \\
\hline & & & \multicolumn{2}{|c|}{$\begin{array}{c}\mathrm{I} \\
\left(30-34.9 \mathrm{~kg} / \mathrm{m}^{2}\right)\end{array}$} & \multicolumn{2}{|c|}{$\begin{array}{c}\text { II } \\
\left(35-39.9 \mathrm{~kg} / \mathrm{m}^{2}\right)\end{array}$} & \multicolumn{2}{|c|}{$\begin{array}{c}I I I I \\
\left(>40 \mathrm{~kg} / \mathrm{m}^{2}\right)\end{array}$} & \\
\hline & $\mathrm{N}$ & $\%$ & $\mathrm{n}$ & $\%$ & $\mathrm{n}$ & $\%$ & $\mathrm{n}$ & $\%$ & \\
\hline Normal & 6 & 18.8 & -- & -- & -- & -- & -- & -- & 6 \\
\hline Tendencia al exceso & 8 & 25.0 & 6 & 18.8 & 2 & 10.5 & -- & & 18 \\
\hline Exceso evidente & 18 & 56.2 & 25 & 80.6 & 17 & 89.5 & 8 & 100 & 69 \\
\hline Total & 32 & 100 & 31 & 100 & 19 & 100 & 8 & 100 & 90 \\
\hline
\end{tabular}




\begin{tabular}{|lcc|}
\hline $\begin{array}{c}\text { Cuadro 5. Distribución de la muestra según los } \\
\text { niveles de triglicéridos* }\end{array}$ \\
\hline Categoría & Frecuencia & Porcentaje \\
\hline Normal $(<150 \mathrm{mg} / \mathrm{dl})$ & 40 & 48.2 \\
Normal alto $(150-199 \mathrm{mg} / \mathrm{dl})$ & 22 & 26.5 \\
Alto $(200-499 \mathrm{mg} / \mathrm{dl})$ & 19 & 22.9 \\
Muy alto $(>500 \mathrm{mg} / \mathrm{dl})$ & 2 & 2.4 \\
\hline
\end{tabular}

* Según los criterios de clasificación de NCEP, 2002

\section{Hipertrigliceridemia}

Los niveles plasmáticos de triglicéridos se presentan en el cuadro 5. De acuerdo con la clasificación de NCEP, 2002, sólo $40(44.4 \%)$ mujeres presentaron niveles normales.

\section{Factores dietarios asociados a riesgo de enfermedad cardiovascular}

El consumo de energía y macronutrientes se muestra en el Cuadro 6. Puede observarse que la ingesta de carbohidratos (52.1\% del VET) alcanza la recomendación del NCEP, 2002, mientras que la ingesta de proteína $(13.8 \%)$ es inferior a lo recomendado. Por otra parte, la ingesta de grasa total (34\%) supera la recomendación que es del 25-30\%, según las guías alimentarias para Costa $\mathrm{Rica}^{18}$, pero no la ingesta promedio de colesterol, que está por debajo del límite de ingesta recomendado.

En el caso de los micronutrientes, específicamente vitamina $\mathrm{A}$ y $\mathrm{C}$, se puede observar que la ingesta promedio es satisfactoria. Sin embargo, el consumo de fibra dietaria está por debajo de las recomendaciones, lo que pone en evidencia un factor dietario de riesgo para múltiples enfermedades.(Cuadro 7)

\section{Discusión}

Esta es la primera investigación realizada en el país que valora el perfil de riesgo cardiovascular en mujeres en edad media con sobrepeso y obesidad. De acuerdo con los resultados obtenidos, solo un $50.6 \%$ de las mujeres presentaban un nivel de colesterol deseable, un $61.3 \%$ un nivel de LDL óptimo o cercano al óptimo, más del $20 \%$ con un HDL colesterol por debajo de lo recomendado por el NCEP, y un $51.8 \%$ con un nivel de triglicéridos sobre el nivel recomendado. En resumen, el perfil lipídico encontrado es clásicamente descrito en personas con sobrepeso, a saber: LDL alto, HDL bajo y triglicéridos altos, ${ }^{19}$ lo cual usualmente se ha correlacionado al estado de resistencia a la insulina como parte del síndrome dismetabólico que presentan estas pacientes. ${ }^{15,20-22}$

A pesar de que el $89.1 \%$ presentó niveles de glicemia normal, el no haber medido niveles de insulinemia impidió la medición de parámetros indicadores de resistencia a la insulina que, sin embargo, pueden estimarse como muy probables por el fenotipo general que presentaron estas mujeres. Mediante la investigación se diagnosticaron 9 mujeres como nuevos casos de intolerancia a los carbohidratos y un caso con Diabetes Mellitus, a pesar de acudir regularmente al EBAIS. Es conocido que la obesidad se asocia con diabetes tipo 2 principalmente, lo cual aumenta la incidencia de infarto del miocardio. ${ }^{15}$ Parte del mayor riesgo de la cardiopatía coronaria que se observa en el diabético se atribuye a la presencia concomitante de otros factores de riesgo, como dislipidemias, hipertensión y obesidad..$^{20} \mathrm{La}$ resistencia a la insulina se asocia con varios factores de riesgo cardiovascular, incluyendo hiperglicemia, dislipidemia, hipertensión, trombofilia y fumado..$^{21,22}$

\begin{tabular}{|lccccc|}
\hline \multicolumn{2}{|c}{ Cuadro 6. Ingesta promedio de energía y nutrientes de la dieta, porcentaje de adecuación y porcentaje } \\
del VET
\end{tabular}

Promedio de consumo de tres días

N.A. No aplica 


\begin{tabular}{|ccccccc|}
\hline \multicolumn{7}{|c|}{ Cuadro 7. Ingesta promedio de micronutrientes de las mujeres de la muestra } \\
\hline Micronutriente & Ingesta promedio & mínimo & máximo & EAR ${ }^{\circ}$ & RDA & RDD \\
\hline Vitamina A (ug) & $1337.4 \pm 1400.6$ & 181.1 & 8285 & 500 & 700 & 500 \\
Vitamina C (mg) & $105.3 \pm 81.1$ & 10.5 & 574.8 & 60 & 75 & 60 \\
Fibra dietética (g) & $14.9 \pm 10.2$ & 0.6 & 86.7 & 25 & $20-30$ & $18-24$ \\
\hline
\end{tabular}

Promedio de consumo de tres días

- Estimated Average Requeriment. Food Nutrition Board, 2004

- Recommended Dietary Allowance. Food Nutrition Board, 1997-2001

Recomendaciones dietéticas diarias. Instituto de Nutrición de Centroamérica y Panamá, 1996.

En vista de la conocida correlación entre dislipidemias e hiperglicemias con factores relacionados con el estilo de vida de las personas (dietas ricas en grasas saturadas, colesterol y baja en fibra, así como sedentarismo), es de vital importancia la vigilancia de estos factores en nuestro sistema de atención primario, de modo que se detecten estos casos y se identifiquen estas pacientes como de mayor riesgo y así ofrecer un monitoreo más frecuente de los factores de riesgo cardiovascular.

Entre los factores asociados al estilo de vida de las personas, un $20 \%$ de las mujeres participantes se declararon fumadoras, lo que constituye un factor de riesgo para la enfermedad cardiovascular. ${ }^{23}$ La relación entre fumado y ECV está dada principalmente por alteraciones en el sistema de coagulación; también produce trastornos en los lípidos plasmáticos aumentando los niveles de LDL colesterol y disminuyendo HDL colesterol. ${ }^{24}$

El hallazgo de que un 33.3\% de la muestra presentara cifras de presión arterial elevadas es muy preocupante, pero congruente con otros estudios que consistentemente encuentran que la hipertensión arterial es una condición frecuentemente subdiagnosticada y subtratada. Hay que recordar que la reducción de 5-6 $\mathrm{mmHg}$ en la presión arterial diastólica, reduce un $20-25 \%$ el riesgo a ECV. ${ }^{25}$

Los factores predisponentes de ECV son aquellos que si están presentes incrementan el efecto de los factores mayores. ${ }^{25}$ Se incluye dentro de estos la presencia de obesidad, la cual era evidente en el $55 \%$ de las mujeres del presente estudio, sin dejar de lado el porcentaje restante diagnosticado con sobrepeso. Aunado a esto, se encuentra la situación de que casi la totalidad presentó obesidad abdominal, la cual ha sido especialmente asociada a alteraciones metabólicas hormonales y de las lipoproteínas, ambas evidentes en un porcentaje de estas mujeres, y que constituyen riesgo para enfermedad cardiovacular. ${ }^{26}$

En el caso de Costa Rica y según las encuestas de nutrición existentes, hay un $45.9 \%$ de obesidad en mujeres de 20 a 44 años y un $75 \%$ en las mujeres de 45 a 59 años de edad, ${ }^{27}$ lo cual plantea un llamando de alerta acerca de la importancia de estudiar los posibles factores que podrían estar condicionando el exceso de peso en la población en general, así como la necesidad de identificar terapias efectivas para la pérdida de peso, ya que los estudios muestran como el IMC y la cardiopatía coronaria tienen una correlación positiva, ${ }^{20,28}$ relacionada con otros factores de riesgo que se observan en individuos obesos: intolerancia a la glucosa, diabetes, hipertensión y dislipidemia. ${ }^{20,15}$

Aproximadamente el $49 \%$ de las mujeres indicó tener historia familiar de ECV, lo cual refleja una fuerte carga genética aterogénica en esta población, que se manifiesta cuando se suman factores ambientales asociados al estilo de vida de los pacientes, lo que los pone en riesgo de padecer de ECV a edad más prematura.

Entre los factores asociados al estilo de vida en este grupo, los niveles bajos de actividad física son una condición que promueve tanto el desarrollo del sobrepeso como el riesgo asociado a otros parámetros dismetabólicos. La actividad física reduce el riesgo de cardiopatía coronaria al retardar aterogénesis, aumentar la vascularidad del miocardio y la fibrinólisis. También modifica otros factores de riesgo al incrementar el colesterol HDL, al mejorar la tolerancia a la glucosa y sensibilidad a la insulina, ayudar en el control de peso y reducir la presión arterial. ${ }^{20}$ Los resultados de esta investigación evidencian que la mayoría de la mujeres son sedentarias, no obstante, el indicador de Paffenbarger quizá no fue el mejor método para establecer el nivel de actividad física, ya que al parecer los sujetos estudiados tienden a sobreestimar la actividad física, por lo que la cifra de sedentarismo podría ser mayor.

Al evaluar los factores asociados a la alimentación, Fung et al, ${ }^{29}$ encontraron que patrones dietarios caracterizados por una alta ingesta de carnes rojas y procesadas, productos lácteos altos en grasas y cereales refinados, están correlacionados positivamente con los niveles de colesterol LDL, homocisteína, insulina en ayunas, péptido $\mathrm{C}$, leptina y proteína $\mathrm{C}$-reactiva y todos estos están relacionados con obesidad y un mayor riesgo a ECV. En el caso de las mujeres 
de este estudio, el consumo de grasa excede las recomendaciones nutricionales. El consumo de grasa tiene gran relevancia por su relación con los lípidos plasmáticos y la obesidad. Las grasas producen hipertrigliceridemia posprandial y un aumento de la concentración de triglicéridos cuando no hay ayuno. ${ }^{30}$

Rodríguez et al, en 1996, ${ }^{6}$ estudiaron los factores aterogénicos de la dieta de los costarricenses y encontraron que la contribución de las grasas al valor energético total fue del 35\%, dato igual a los resultados de esta investigación, y la ingesta de ácidos grasos saturados, mono y polinsaturados no corresponde a la recomendación del NCEP, 2002, mientras que el consumo de fibra fue muy bajo (14.9 \pm 10.2 gramos por día).

Es conocido que uno de los factores protectores del la dieta es el consumo de fibra. Se sabe que las dietas ricas en fibra producen descensos de la concentración plasmática de colesterol, sobre todo de LDL, sin que haya variaciones sobre las HDL y los triglicéridos. Estos descensos se han relacionado con un aumento en la excreción de ácido biliar y la síntesis hepática de colesterol. ${ }^{20,31-34}$

A pesar de que el promedio de ingesta para la energía, carbohidratos y proteína no excede las recomendaciones nutricionales, es importante destacar la gran desviación estándar de los resultados, evidenciando un grupo importante de mujeres que no cumplen con dichas recomendaciones. No hay que obviar que los individuos obesos tienden a subestimar el consumo de alimentos, lo cual probablemente introduce un porcentaje de error en la estimación de la ingesta de energía y nutrientes.

Los antioxidantes desempeñan un papel importante en inhibir la oxidación de las lipoproteínas LDL. Existe evidencia de que los procesos oxidativos tienen un rol muy importante en el proceso de la formación de placa aterosclerótica, principalmente por la oxidación de las lipoproteínas de baja densidad. ${ }^{35-37}$ La ingesta de vitamina A y C de estas mujeres satisface las recomendaciones nutricionales, sin embargo, queda por investigar la ingesta promedio de vitamina $\mathrm{E}$ (importante antioxidante) y promover dietas saludables, de modo que se aseguren la ingesta adecuada de estos antioxidantes, sobre todo al observar la variación entre los resultados.

Luego del análisis respectivo de los diferentes factores de riesgo de enfermedad cardiovascular evaluados en este grupo de mujeres con sobrepeso y obesidad, se concluye que los factores de riesgo mayores de enfermedad cardiovascular más prevalentes fueron el LDL colesterol elevado, el colesterol total elevado y la hipertensión, mientras que los factores de riesgo predisponentes más prevalentes fueron la obesidad y el sedentarismo. El único factor de riesgo condicionante que se evaluó fue la hipertrigliceridemia, presentada en el $26.3 \%$ de las mujeres.
Puede considerarse a estas mujeres en edad media como de riesgo elevado para enfermedad cardiovascular arterioesclerótica. Tomando en cuenta que se trata de una población joven que, además, tiene usualmente a su cargo la mayor parte de las responsabilidades familiares, tanto social, educativa $\mathrm{y}$, cada vez más frecuentemente, económica, se puede considerar el impacto que sobre este grupo poblacional tiene alta morbi-mortalidad asociada al elevado riesgo cardiovascular. Los resultados del presente estudio han de servir para llamar la atención con el fin de que se implementen acciones específicamente dirigidas a la mujer en su edad media con sobrepeso, como un propio riesgo especialmente elevado de enfermedad cardiovascular y sus complicaciones.

Agradecimientos: esta investigación no hubiese sido posible sin la participación de los personeros del PAIS, el apoyo de la Vicerrectoría de Investigación de la Universidad de Costa Rica y el aporte de la Compañía Dos Pinos.

\section{Referencias}

1. World Health Organization (WHO).Types of cardiovascular disease. En:http://www.who.int/cardiovascular_diseases/en/cvd atlas 01 types.pdf. Consultado el 12 de septiembre de 2005

2. Ministerio de Salud. Memoria anual de 1993. Costa Rica: Departamento de publicaciones e impresos. 1994.

3. Organización Panamericana de la Salud [OPS] .Informe Salud de las Américas Costa Rica 2002. Consultado en: http//www.paho.org/. 2001. Citado el $1^{\circ}$ de noviembre del 2005.

4. Meza, N. Hábitos alimentarios de la población costarricense. Programa CEN-CINAI. Seminario "índice glicémico en salud y alimentación humana". INCIENSA: Costa Rica. 2002.

5. American Herat Association (AHA). Risk Factors and Coronary Herat Disease AHA Scientific Position.

En: http://www.americanheat.org/presenter.jhtml?identifier=4726.2000. Consultado el 7 de marzo de 2006

6. Rodríguez N, Arauz A, Meza N, Rosell M. Factores aterogénicos de la dieta de la población costarricense, 1991. Arch Latinoam Nutr 1996; 46: 27-32.

7. Ministerio de Salud. Encuesta Nacional de Nutrición. Fascículo 1 Antropometría. Ministerio de Salud, INICIENSA. San José, Costa Rica. 1996.

8. Baylin A, Kabagambe E, Ascherio A, Spigelman D, Campos H. High 18:2 Trans-fatty acids in adipose tissue are associated with increased risk of nonfatal acute myocardial infarction in Costa Rican adults. J Nutr 2003;133: 1186-1191.

9. Roselló M, Guzmán S. Trends in mortality from ischemic herat disease and acute myocardial infarction in Costa Rica, 1970-2001. Rev Panam Salud Publica 2004; 16: 295-301.

10. Ministerio de Salud. Encuesta basal de factores de riesgo de enfermedades no transmisibles Cartago 2001. Modulo 1: Factores alimentario nutricionales. $1^{\circ}$ ed. San José, Costa Rica. 2003.114p.

11. Paffenbarger R, Wing A, Hyde R. Physical activity as an index of heart and attack risk in college in alumni. Am J Epidemiol 1978; 108: 161-175.

12. Paffenbarger RS, Blair S.N, Lee I-M, Hyde R.T. Measurement of physical activity to asses health effects in free-living populations. Med Sci Sports Exerc 1993 ; 25: 60-70. 
13. Chinnok, A. Diario de consumo de alimentos: Instrumento para el registro de información. Universidad de Costa Rica. Escuela de Nutrición. San José, Costa Rica. 2005

14. American Diabetes Assosiation(ADA). Using the Diabetes Food Pyramid. En: http://www.diabetes.org/nutrition-and-recipes/nutrition/ foodpyramid.jsp. 2005. Consultado el 28 de junio de 2006

15. National Colesterol Program (NCEP). Evaluation, and treatment of high blood cholesterol in adults (Adult Treatment Panel 11l) final report. United States: NIH Publication. 2002; 02: 5215.

16. Frisancho, R. New norms of upper limb fat and muscle areas for assessment of nutritional status. Am. J Clin Nutr 1981; 34: 25402545.

17. Organización Mundial de la Salud (OMS). La obesidad en la pobreza. Consultado en:_http://www.paho.2000. Citado el 20 de agosto de 2005.

18. Ministerio de Salud. Guías alimentarias para la educación nutricional en Costa Rica. $3^{\circ}$ edición. San José, Costa Rica. 2001

19. Jellinger P, Dickey R, Ganda O, Mehta, Nguyen T, Rodbard H., et al. The american association of clinical endocrinologists medical guidelines for clinical practice for the diagnosis and treatment of dyslipidemia and prevention of atherogenesis. Endocrine Practice 2000; 6: 162-213.

20. Krummel D. Nutrición en las enfermedades cardiovasculares. En Mahan, K \& Scott-Stump, S (Eds.), Nutrición y Dietoterapia de Krause. $9^{\text {na }}$ edición (pp.607-646). México, D.F.: Mc Graw Hill.2001

21. Bonora E, Formentini G, Calcaterra F, Lombardi S, Marini F, Zenari $\mathrm{L}$, et al. HOMA-estimated insulin resistent is an independent predictor of cardiovascular disease in type 2 diabetic subjects. Diabetes Care 2002; 25: 1135-1141.

22. Wallace T, Levy J.C., Mathews D.R. Use and abuse of HOMA modeling. Diabetes Care 2004; 27: 1487-149.

23. Grundy S, Balady G, Criqui M, Fletcher G, Greenland P, Hiratzka L. Primary prevention of coronary heart disease: guidance from Framingham. A statement for heathcare professionals from the AHA task force on risk reduction. Circulation 1998; 97: 1876-1887.

24. Steiner G, Schwartz L, Shumak S, Poapst M. The association of increased levels of intermediate- density lipoproteins with smoking and with coronary artery disease. Circulation 1987; 75: 124-130.

25. Grundy S, Balady G, Criqui M, Fletcher G, Greenland P, Hiratzka L. Primary prevention conference V. Beyond secondary prevention: identifying the high risk patient for primary prevention: medical office assesment: writing group I. Circulation 2000; 101: e3-e11.
26. Suk Sh, Sacco RL, Boden-Albala B, Cheun JF, Pitmman JG, Elkind MS, et al. Abdominal obesity and risk of ischemic stroke. Stroke 2003;34: 1586-1592.

27. Cheun JF, Pittman JG, Elkind MS, Paik MC. Abdominal obesity and risk of ischemic stroke, the northern Manhattan. Stroke study. Stroke 2003; 34: 1586-1592.

28. Zhou B, Wu Y, Yang J, Li Y, Zhang H, Zhao L. Overweight is an idependent risk factor for cardiovascular disease in China populations. The international Association for the study of obesity. Obes Rev 2002; 3: $147-156$

29. Fung T. Rimm E.B, SpiegeIman D, Rifai N, Tofler GH, Wilett WC. et al. Association between dietary paterns and plasma biomakers of obesity and cardiovascular disease risk . Am J Clin Nutr 2001;73: 6167.

30. Jacobsen MU, Overvard K, Dyerberg J, Schroll M, Heitmann B. L. Dietary fat and risk of coronary heart disease: Possiblle effect modification by gender and age. Am J Epidemiol 2004; 160: 141149.

31. Cervera P, Clapes J, Rigolfas R. Alimentación y dietoterapia. España: Mc Graww Hill. 1999.

32. Wolk A, Manson JE, Stampfer MJ, Colditz GA, Hu FB, Speizer FE et al. Long term intake of dietary fiber and decreased risk of coronary heart disease among women. JAMA 1999; 281:1998-2004.

33. Wu H, Dwyer K, Fan Z, Shicore A, Fan J, Dwyer J. Dietary fiber and progression risk factor for atherosclerosis: The Los Angeles atherosclerosis study. Am J Clin Nutr 2003; 78:1085-1091.

34. Nicolosi RJ, Wilson TA, Lawton C, Handleman GJ. Dietary effects on cardiovascular disease risk factors: beyond saturated fatty acids and cholesterol. J Am Coll Nutr 2001;20: 421S-427S.

35. Jha P, Flather M, Lonn E, Farkouh M, Yusuf S. The antioxidant vitamins and cardiovascular disease. Ann Intern Med 1995; 123:860872.

36. Osganian S, Stampfer M, Rimm, Spiegelman D, Manson J, Willet, W Dietary carotenoids and risk of coronary artery disease in women. A J Clin Nutr 2003; 77: 1390-1399.

37. Tribble D. Antioxidant consumption and risk of coronary disease: emphasis on vitamin $\mathrm{C}$, vitamin $\mathrm{E}$, and $\beta$-carotene: a statement for healthcare professionals from the american heart association. Circulation 1999; 99:591-595. 